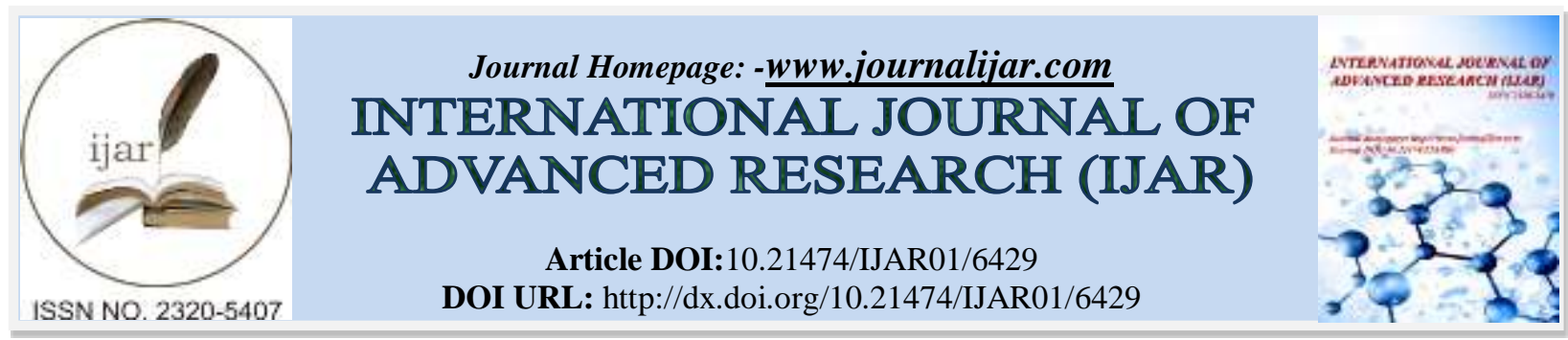

RESEARCH ARTICLE

\title{
EFFECTS ON THE PROPERTIES OF LOW DENSITY POLYETHYLENE COMPOSITES REINFORCED WITH TREATED AND UNTREATED BETEL NUT HUSK FIBERS.
}

Tanvir Sultana ${ }^{1}$, Shahin Sultana ${ }^{2}$, Husna P. Nur ${ }^{2}$ and Md. Wahab Khan ${ }^{1}$.

1. Department of Chemistry, Bangladesh University of Engineering and Technology (BUET), Dhaka.

2. Fiber \& Polymer Research Division, BCSIR Laboratories Dhaka, BCSIR, Dhaka-1205, Bangladesh.

\section{Manuscript Info}

\section{Manuscript History}

Received: 03 December 2017

Final Accepted: 05 January 2018

Published: February 2018

Keywords:-

Betel nut husk fiber,LDPE, composite, tensile strength, water absorption etc.

\begin{abstract}
Betel nut (Areca catechu) husk fibers (BNHF) were extracted and used to prepare sodium hydroxide treated and untreated BNHF reinforced low density polyethylene (LDPE) composites. Extracted and washed BNHF were treated with sodium hydroxide to improve their adhesion to LDPE matrices. Chemical treatment of betel nut husk fibers with sodium hydroxide was carried out and the change in the functional group of treated BNHF was studied by FT-IR spectroscopic analyses. Sodium hydroxide treated and untreated BNHF were used in this case of research as reinforcing materials to find out their effects on LDPE composites. Different types of compression molded BNHF-LDPE composite were prepared using different weight percent of treated and untreated BNHF with compositions 5, 15, 20, 25 and $30 \mathrm{wt} \%$ in LDPE. The tensile, water absorption and morphological properties were studied for all composites. Enhancement in the tensile, water absorption and morphological properties were shown in the treated BNHF -LDPE composites. Alkaline treatment reduced the water absorption and increased the tensile properties of treated betel nut husk fiber-LDPE composites. Morphological analyses showed the evidence of stronger interfacial adhesion between the fibers and matrices in the alkali treated BNHF-LDPE composites than the untreated BNHF-LDPE composites.
\end{abstract}

Copy Right, IJAR, 2018,. All rights reserved.

\section{Introduction:-}

Over the last few years, as a result of the growing environmental awareness about greenhouse gas emissions and environmental pollution, manufacturers and scientists are keen to study novel environmental friendly materials. Intensive research and developments have been carried out by many researchers, engineers and scientists to develop biodegradable and sustainable composites using natural fibers ${ }^{(1)}$. Synthetic fibers like carbon and glass though exhibits excellent mechanical, thermal properties and durability but difficulties in disposal processing which produce lot of black smoke and bad odors. In order to overcome this problem, necessity of natural fibers based composites is demanding to make the world 'green'. The composites referred to as bio-composites which are combine natural fibers such as kenaf, jute, hemp and sisal with biodegradable or non-biodegradable polymers ${ }^{(2)}$. Natural fibers are cheaper and substitute for synthetic fibers. Compared with synthetic fibers, natural fibers have many advantages like low density, cheaper, high strength, high stiffness, no harm to environment, renewable sources and biodegradable properties ${ }^{(3)}$. 
In this research work, betel nut husk fibers were used as reinforcing materials with thermoplastics to make composites. Betel/ Areca nut is the seed of Areca palm and Areca catechu is a species of palm which grows mainly in the Southeast Asia and South Asia region. It is abundantly available in Bangladesh as waste fibers of betel nut fruits. Composites made with natural fibers offers use in various applications such as aerospace, leisure, construction, households, sport, packaging, automotive industries by replacing metallic components due to their inherent light weight and immune to corrosion ${ }^{(2,4)}$. However due to hydrophilic nature of natural fibers give rise to incompatibility between fiber and polymer in composites. This leads to undesirable properties of the composites. Hence fiber surface is modified with coupling agent in order to have interfacial adhesion between fiber and matrix ${ }^{(2)}$. Natural fibers are composed of cellulose, hemicelluloses, lignin, pectin, wax, and ash. Chemical treatment of the natural fibers can overcome the limitations due to hydrophilic nature by cleaning the fiber surface, chemically modify the surface, stop the moisture absorption process and increase the surface roughness ${ }^{(5)}$. Swain et al. worked on abrasive wear behavior of surface modified jute fiber reinforced epoxy composite ${ }^{(6)}$. They reported that benzoyl chloride treated based composite notably gave better abrasive wear resistance than the untreated and alkali treated based composite. This was due to the high-interfacial adhesion and low-composite porosity. Siregar et al. investigated and reported on effect of the alkali $(\mathrm{NaOH})$ treatment on the mechanical properties of pineapple leaf fiber (PALF) reinforced high impact polystyrene (HIPS) composites ${ }^{(7)}$. They obtained highest mechanical properties value for the short PALF treatment with $\mathrm{NaOH}$ concentration of $4 \%$. Prasad et al. investigated on effect of the alkali $(\mathrm{NaOH})$ treatment followed by bleaching with $\mathrm{H}_{2} \mathrm{O}_{2}$ on tensile properties of short jute fiber reinforced polypropylene and reported about small increase in the strength of composite with treated fibers compared to composite with untreated fibers ${ }^{(8)}$. In a review by Ram et al., it is shown that some works were done in the field of replacing existing industrial safety helmet material acrylonitrile butadiene styrene (ABS), with variety of polymer composites $^{(9)}$. The study shows that hybrid polymer composites have good potential to replace ABS in safety helmets. Desai et al. also reported in a review on effectiveness of Areca (betel) fiber as a reinforcing material in ecofriendly composites ${ }^{(10)}$. The review report states that in the recent past, it has been noticed that not much appreciable research been carried out on the optimized surface treatment, manufacturing techniques, and product application using the areca fibers. Hence, there are more opportunities to develop economical and ecologically superior engineered material by utilizing these areca fibers and its composites.

In this piece of research work, betel nut husk fibers were treated with sodium hydroxide to improve its adhesion with LDPE. The aim of this research is to find out the effects of treated and untreated BNHF loading on the physicomechanical properties of betel nut husk fiber-LDPE composites.

\section{Experimental Part:- Materials:-}

Low density polyethylene (LDPE) was purchased from Polyolefin Company Pvt. Ltd., Singapore. Melting point of this LDPE was found to be $115^{\circ} \mathrm{C}$. Betel nuts were collected from Barisal, Bangladesh and the BNHF were extracted from these betel nuts. Sodium hydroxide was collected from Merck, Germany.

\section{Processing of betel nut husk fibers:-}

Betel nut husk fibers were extracted from collected betel nuts. The fibers were cutted into 1 to $3 \mathrm{~mm}$ pieces. Fibers were sieved and dried after washing.

\section{Surface modification of betel nut husk fibers(BNHF) by sodium hydroxide:-}

Aqueous solution of sodium hydroxide (6\%) was prepared to conduct reaction with BNHF. Dried betel nut husk fibers were immersed in that solution for one hour at room temperature approximately $25^{\circ} \mathrm{C}$ and the fibers were stirred occasionally with a glass rod. Fiber to liquor ratio was 1:20 (w/v). The alkali treated BNHF were thoroughly washed in tap water and then immersed in distilled water for $24 \mathrm{hrs.} \mathrm{Finally,} \mathrm{fibers} \mathrm{were} \mathrm{washed} \mathrm{with} \mathrm{distilled} \mathrm{water}$ to remove the alkali completely and the $\mathrm{pH}$ was measured until it was found neutral. The alkali treated BNHF were dried in air first then further dried in an oven to get a constant weight.

\section{FT-IR spectroscopic characterization of untreated and treated betel nut husk fibers:-}

The infrared spectra of untreated and treated BNHF were recorded on a FT-IR/NIR Spectrometer (Forntier, Perkin Elmer, USA). To take FT-IR, the treated and untreated betel nut husk fibers were used to make pellet with potassium bromide. Approximately $0.5 \mathrm{mg}$ of dried and powdered BNHF was mixed with approximately $100 \mathrm{mg}$ of dried powdered potassium bromide in a small agate mortar pestle. The mixture was taken in a die to make pellets by applying vacuum pressure. IR spectra were obtained in the printed form. 
Fabrication of composites by compression moulding technique:-

Before fabrication of composites treated and untreated betel nut husk fibers were dried in an oven at $80{ }^{\circ} \mathrm{C}$ for 24 hours. LDPE was granulated in a grinder and dried at $80{ }^{\circ} \mathrm{C}$ for 3 hours.

The LDPE and BNHF were dry-blended in a blender for one minute. These mixers were then moulded in a compression moulding machine (Paul-Otto Weber Press Machine) at a molding temperature of $150{ }^{\circ} \mathrm{C}$. These procedures were followed for all the composites fabricated in the ratio of composition shown in the Table I:

Table 1:-The composition of treated and untreated betel nut husk fibers (\%) and LDPE polymer matrices (\%) in composites

\begin{tabular}{|c|c|}
\hline Treated or untreated betel nut husk fibers (\%) & $\begin{array}{c}\text { Polymer Matrices } \\
\text { LDPE (\%) }\end{array}$ \\
\hline 0 & 100 \\
\hline 5 & 95 \\
\hline 10 & 90 \\
\hline 15 & 85 \\
\hline 20 & 80 \\
\hline 25 & 75 \\
\hline 30 & 70 \\
\hline
\end{tabular}

The mixtures were hot pressed for 10 minutes and the applied pressure was $200 \mathrm{kN}$. The additional pressure of 50 $\mathrm{kN}$ was applied to get voids free compression moulded composite. The composite was allowed to cool and then completely cured composite was taken out from the mould. The same conditions of time, temperature, heating time, pressure and cooling time were maintained to prepare all composites. Finally, the compression moulded composites were cut to make specimens of suitable dimension to measure tensile and water absorption properties.

Water absorption test of composites:-

Sodium hydroxide treated and untreated betel nut husk fiber-LDPE composites were used to conduct water absorption tests. The dimension of each test specimens were $39 \mathrm{~mm}$ x $10 \mathrm{~mm} \times 4 \mathrm{~mm}$ and the testing was carried out following ASTM D 570-99 standard method. The composite specimens were immersed in boiling distilled water bath according to ASTM D 570-99 standard method. Three specimens were taken to calculate average results and presented in the results and discussion section.

Tensile properties of the composites:-

All the treated and untreated betel nut husk fiber-LDPE composites were used to measure the tensile properties of the composites using an universal tensile testing machine, model: 1410 Titans, capacity: $5 \mathrm{kN}$, England. Tensile tests were conducted following ASTM D 3039/D 3039 M-00 (2002) standard method and the cross head speed of the test was $10 \mathrm{~mm} / \mathrm{min}$. Each test of the specimen was performed until tensile failure occurred except $100 \%$ LDPE composite. Six to ten specimens of each composition were tested and the average values were reported by calculating maximum five values.

\section{Results and Discussion:-}

FTIR spectroscopic characterization of untreated and alkali treated betel nut husk fibers:-

The FTIR spectra of untreated and alkali treated BNHF are shown in figure 1. Sodium hydroxide treatment was done to improve the adhesion between fiber and matrix in the composites. The characteristic bands of untreated and alkali treated BNHF are summarized in table II and table III respectively. It is clear from the figures that the absorption band at $1738.37 \mathrm{~cm}^{-1}$ of untreated BNHF have disappeared completely in the treated BNHF. This indicates that the alkali treatment of betel nut husk fibers had removed the impurities, hemicelluloses and part of lignin. The similar FT-IR results were also reported ${ }^{11}$ by Oushabi et al in the case of alkali treated date palm fibers. 
Table II:-FTIR spectral data of untreated raw betel nut husk fiber

\begin{tabular}{|c|c|}
\hline Position of bands $\left(\mathrm{cm}^{-1}\right)$ & Peak assignments \\
\hline$\sim 3422.40$ & $-\mathrm{OH}$ stretching from the cellulose, hemicellulose and lignin \\
\hline$\sim 2919.57$ & $\mathrm{C}-\mathrm{H}$ vibration in aromatic ring and alkanes \\
\hline$\sim 1738.37$ & $\mathrm{C}=\mathrm{O}$ stretching from the lignin and hemicellulose \\
\hline$\sim 1610.31$ & $\mathrm{C}=\mathrm{C}$ stretching from aromatic in-plane \\
\hline$\sim 1513.87$ & $\mathrm{C}=\mathrm{C}$ stretching in aromatic skeletal ring due to lignin \\
\hline$\sim 1428.43$ & $\mathrm{C}-\mathrm{C}$ stretching from aromatic ring \\
\hline
\end{tabular}

Table III:-FTIR spectral data of sodium hydroxide treated raw betel nut husk fiber

\begin{tabular}{|c|c|}
\hline Position of bands $\left(\mathrm{cm}^{-1}\right)$ & Peak assignments \\
\hline$\sim 3423.26$ & $-\mathrm{OH}$ stretching from the cellulose, hemicellulose and lignin \\
\hline$\sim 2922.18$ & $\mathrm{C}-\mathrm{H}$ vibration in aromatic ring and alkanes \\
\hline$\sim 1609.86$ & $\mathrm{C}=\mathrm{C}$ stretching from aromatic in-plane \\
\hline & $\mathrm{C}=\mathrm{C}$ stretching in aromatic skeletal ring due to lignin \\
\hline & C-C stretching from aromatic ring \\
\hline
\end{tabular}

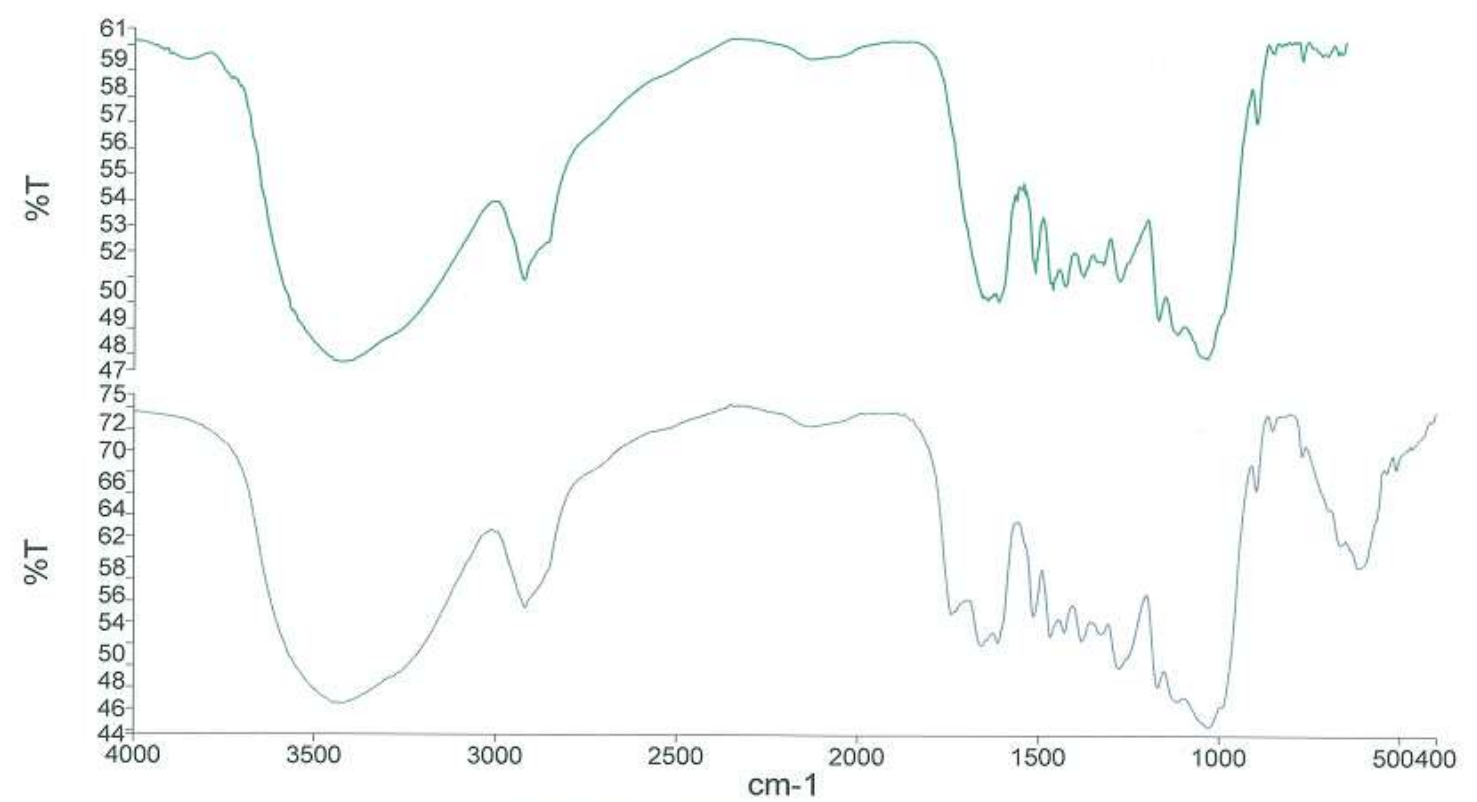

Fig 1:- FTIR spectra of untreated betel nut husk fiber (lower) and sodium hydroxide treated betel nut husk fiber (upper)

Tensile properties of untreated and treated betel nut husk fiber reinforced low density polyethylene (LDPE) composites:-

Tensile strength and elongation at break:-

The tensile strengths of the untreated and treated BNHF-LDPE composites are shown in figure 2. It is observed from the figure that the tensile strengths of the composites increased up to $10 \mathrm{wt} \%$ fibers loading \& then decreased. The elongations at break of untreated and treated BNHF-LDPE composites are shown in figure 3. Elongation at break of all composites decreases with increasing fiber loading (Fig.3). Tensile properties of all treated BNHF-LDPE composites are higher than that of untreated BNHF-LDPE composites. The tensile strength and ductility were better in the case of BNHF-LDPE composites than that of untreated BNHF-LDPE composites. This may be the reason for the improvement of the fiber-matrix interfacial adhesion in composites made by alkali treatment. 


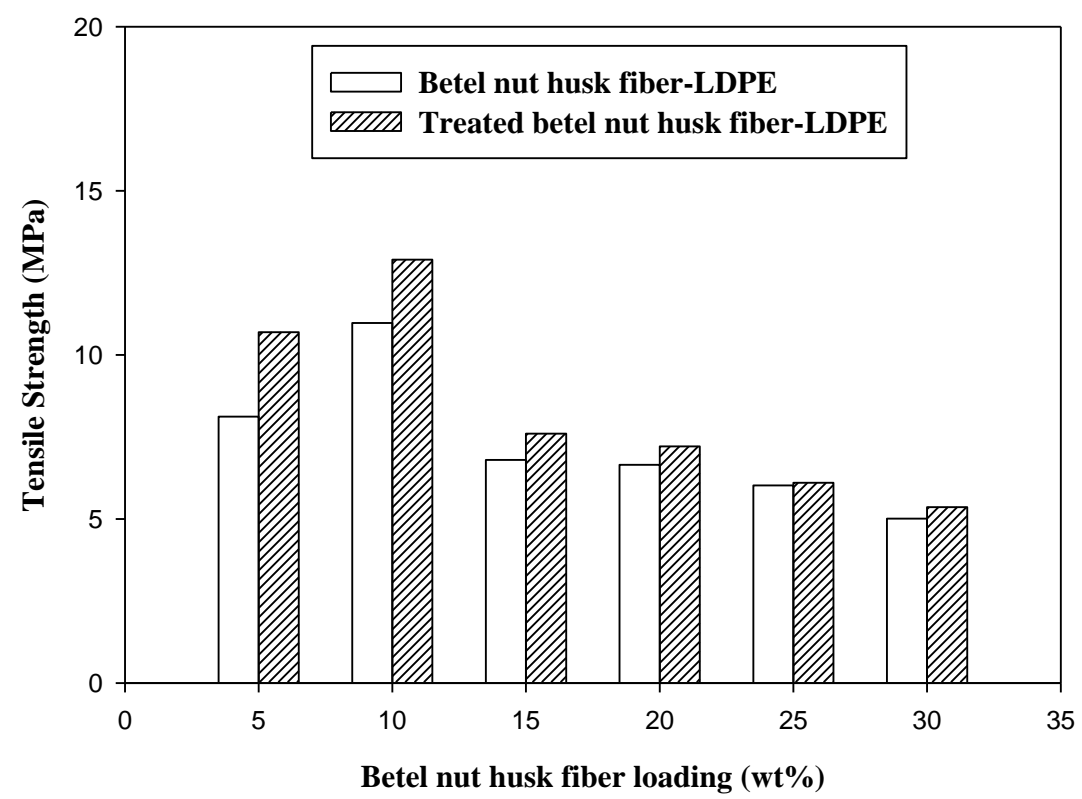

Fig 2:- Tensile strength (MPa) vs betel nut husk fiber (wt\%) curves for raw \& treated betel nut husk fiber-LDPE composites

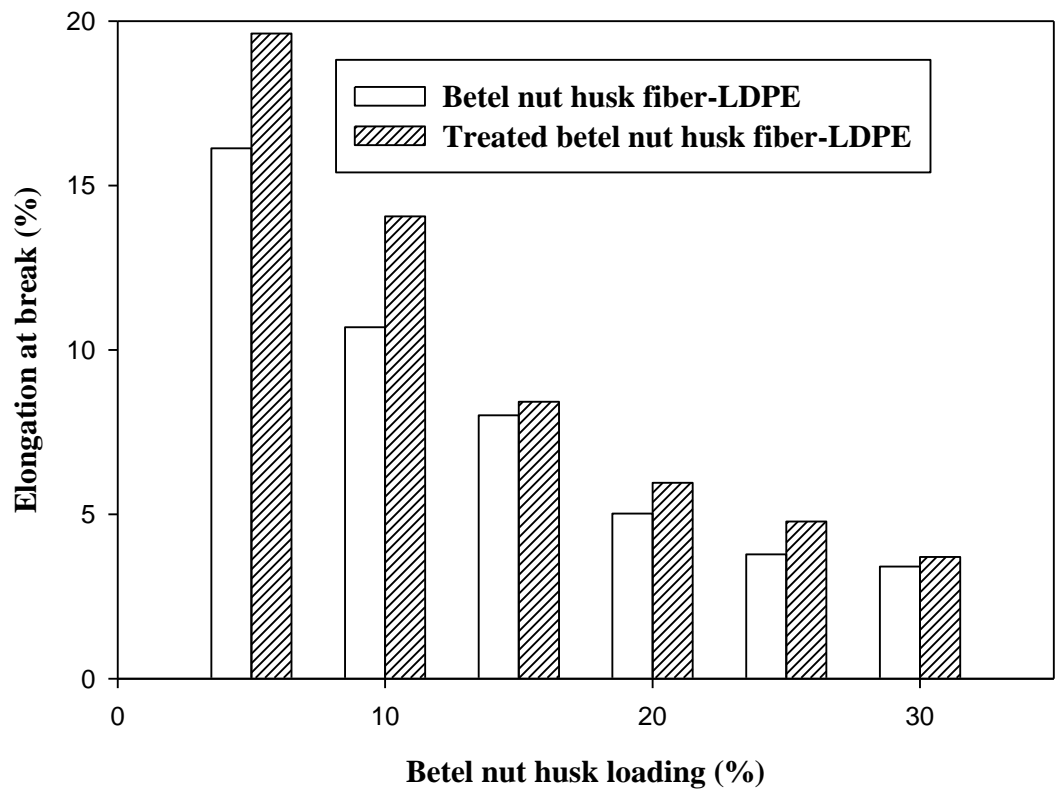

Fig 3:- Elongation at break $(\%)$ vs betel nut husk loading $(\%)$ curves for raw \& treated betel nut husk-LDPE composites

\section{Water absorption:-}

Water absorption test results of all treated and untreated BNHF-LDPE composites are shown in Fig.4. It is clear from the figure that water absorption increases with increasing fiber loading for all composites but water absorption of untreated BNHF-LDPE composites is higher than that of treated betel nut husk fiber-LDPE composites. This may be due to the hydrogen bonding formation of water molecules with the hydroxyl groups in the constituents of lignin, 
hemicelluloses and cellulose of untreated BNHF. Alkali treatment of BNHF removes their hemicelluloses completely and reduces the lignin part. So treated betel nut husk fiber-LDPE composites show less water absorption than untreated BNHF-LDPE composites. So dimension stability will be higher for treated betel nut husk fiber-LDPE composites than untreated BNHF-LDPE composites.

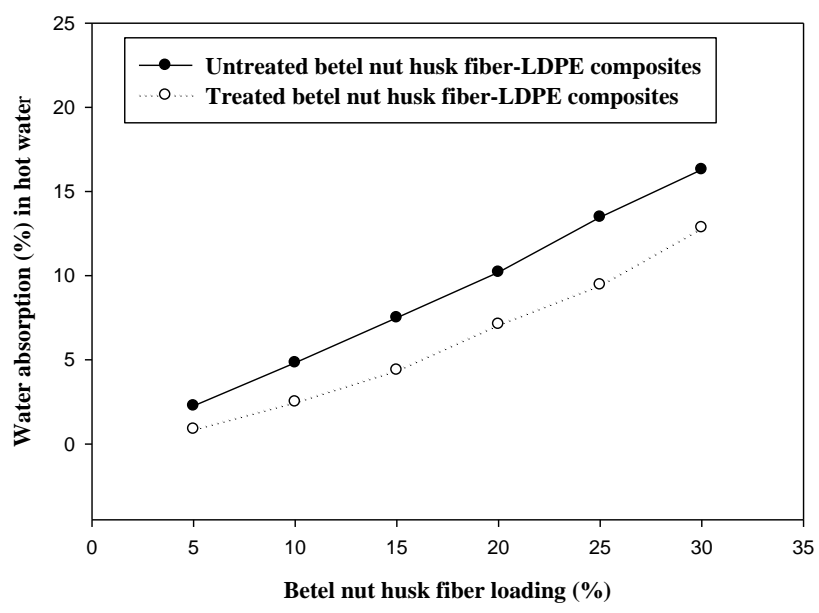

Fig 4:- Hot water absorption (\%) vs fiber loading (\%) curves for untreated and treated betel nut husk fiber-LDPE composites

\section{Morphological analyses:-}

The Scanning electron microscopy (SEM) images of treated and untreated betel nut husk fiber-LDPE composites are shown in figure 5 and 6 respectively. The SEM Micrograph of fracture surfaces of 25 wt- $\%$ alkali treated BNHFLDPE composite shows uniform dispersion and better interfacial adhesion between the fiber and matrix in figure 6 . The weak interfacial adhesion between the fiber and matrix is observed in figure 5 which represents the fracture surface of $25 \mathrm{wt}-\%$ untreated BNHF-LDPE composites. So it may be concluded that alkali treated fibers are able to improve the compatibility between the fiber and matrix in the composites.

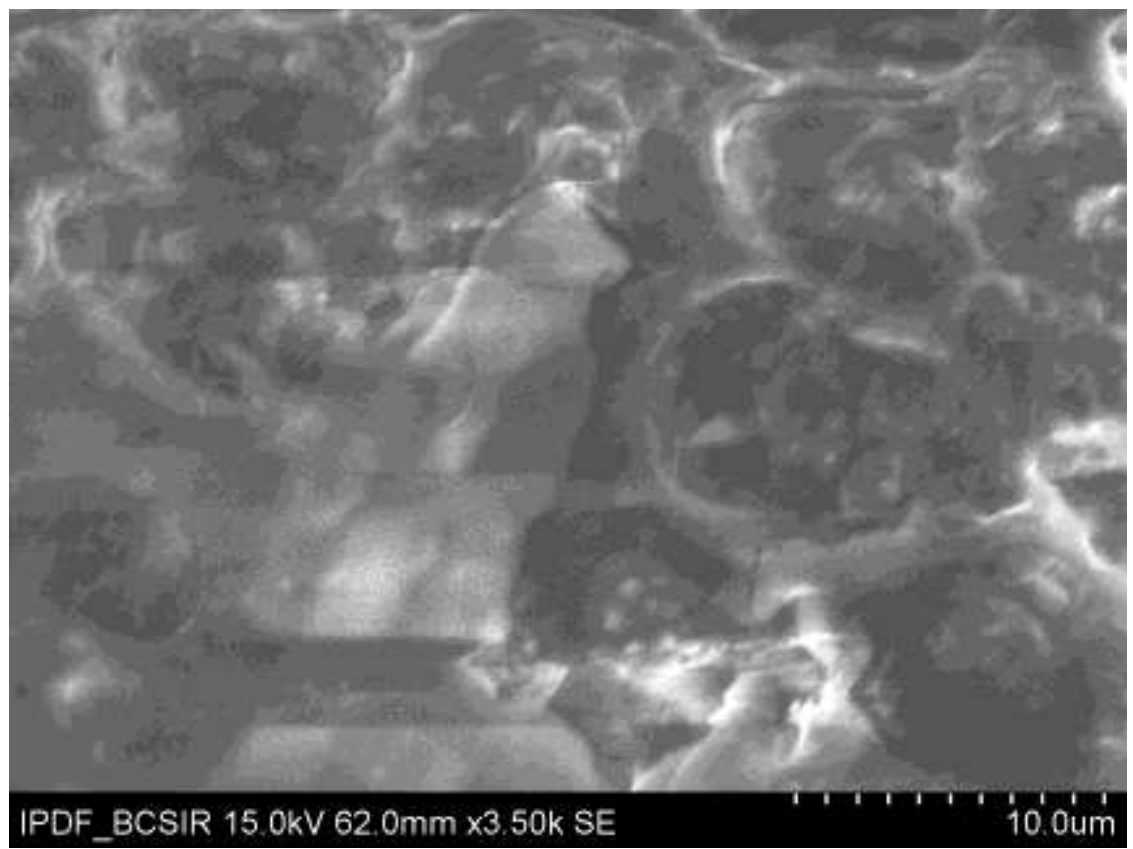

Fig 5:- SEM image of untreated 25 wt-\% betel nut husk fiber-LDPE composite 


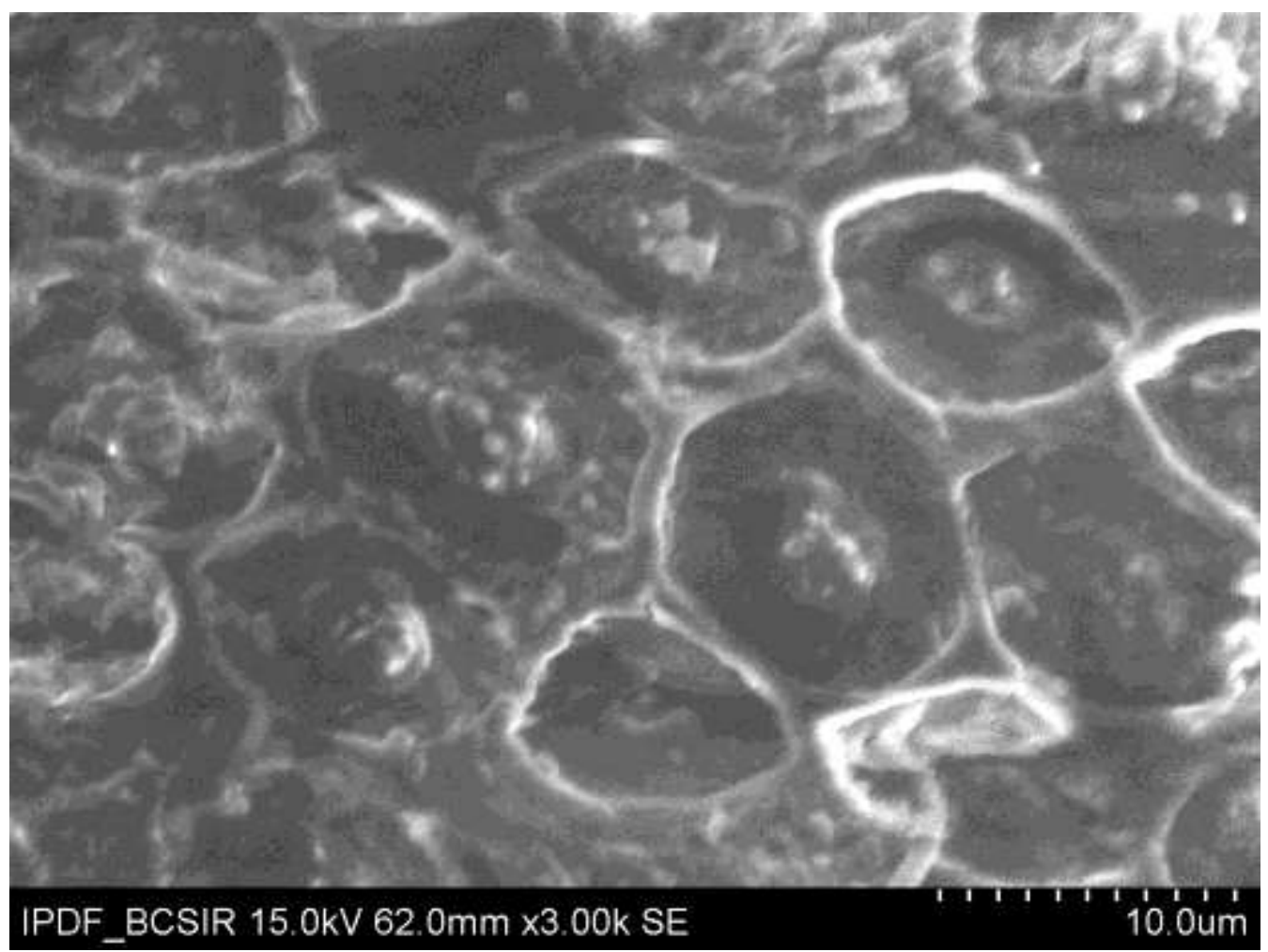

Fig 6:- SEM image of alkali treated $25 \mathrm{wt}-\%$ betel nut husk fiber-LDPE composite

\section{Conclusion:-}

The treatment of betel nut husk fibers with $\mathrm{NaOH}(6 \%)$ removes the impurities and hemicelluloses and reduces the lignin content of fibers. FT-IR spectroscopic analyses also supported the evidence of removal of hemicelluloses. Alkali treatment of BNHF reduces the moisture absorption property and improves the interfacial adhesion between fibers and matrices in composites. The enhancement of the hydrophobicity in BNHF due to alkali treatment results the better compatibility between the fibers and matrices of the LDPE composites. The tensile properties of treated BNHF -LDPE composites are higher than that of untreated betel nut husk fiber-LDPE composites. The reduction of water absorption in the alkali treated betel nut husk fiber-LDPE composites will increase their dimensional stability. The morphological observation also proves the strong interfacial bonding between fibers and matrices in the alkali treated BNHF-LDPE composites. So this research may provide valuable contributions to developing betel nut husk fibers as sustainable reinforcements in thermoplastic based composites.

\section{Acknowledgements:-}

The authors express their sincere gratitude to the BUET, UGC, Ministry of Education and BCSIR Laboratories, Dhaka for supporting the research work. Special thanks to BCSIR Laboratories, Dhaka for providing different useful instruments along with other lab facilities. They are also thankful to Mohammad Shahjahan, Scientific Officer, Md. Joynal Abedin, Scientific Officer and Zahidul Islam, Research Chemist of BCSIR Laboratories, Dhaka for their sincere helping attitude during experimental works. 


\section{References:-}

1. J. Zhu., H. Zhu., J. Njuguna. \& H. Abhyankar., Materials., 6, P. 5171-5198 (2013).

2. P. Gaikwad., P. Mahanwar., \& V. Bambole., International Journal of Chemical, Environmental \& Biological Sciences (IJCEBS)., 2 (4), P.181-186, (2014).

3. P. S. Latha., \& M. V. Rao., International Journal of Innovative Research in Science,Engineering and Technology.,6 (2), P. 2438-2446 (2017).

4. P. Gopal., \& V. K. B. Raja., Research Journal of Pharmaceutical, Biological and Chemical Sciences., 8(2), P. 457-461 (2017).

5. A. Gupta., \& A. Kumar., Asian Journal of Chemistry., 24(4), P. 1831-1836 (2012).

6. P. T. R. Swain., \& S. Biswas., Materials Research., 20 (3), P. 661-674 (2017).

7. J. P. Siregar., S. M. Sapuan., M. Z. A. Rahman. \& H. M. D. K. Zaman., Journal of Food, Agriculture \& Environment., 8 (2), P. 1103 - 1108 (2010).

8. G. L. S. R. Prasad., M. V. H. S. Kumar., \& G. Rajesh., International Journal of Engineering Science Invention., 3 (3), P. 30-34 (2014).

9. K. Ram, V. Chaudhary., F. Ahmad., \& P. K. Bajpai., International Journal of Advanced Production and Industrial Engineering., 506, P. 34-38 (2017)

10. R. H. Desai., L. Krishnamurthy., \&T. N. Shridhar., Indian Journal of Advances in Chemical Science.,S1, P. 27-33 (2016).

11. A. Oushabi., S. Sair., F. O. Hassani., Y. Abboud., \& O. Tanane., South African Journal of Chemical Engineering., 23, P. 116-123 (2017). 